\title{
Reservoir characteristics of Chang 2 Member of Yanchang Formation in Area A, Ordos Basin
}

\author{
Yajie Jing ${ }^{1}$, Zhiwu Zhang ${ }^{1}$, Peng $\mathrm{Xu}^{1}$ and Shasha Yang ${ }^{2, *}$ \\ ${ }^{1}$ Zhidan Oil Production Plant, Shaanxi Yanchang Petroleum (Group) Co. Ltd., Yan'an, Shaanxi, 716000, China \\ ${ }^{2}$ Shaanxi Key Laboratory of Safety and Durability of Concrete Structures, XiJing University, Xi'an, Shaanxi, 710123, China
}

\begin{abstract}
With the continuous enhancement of exploration and development in the Ordos Basin, in-depth research has been carried out on the petrological and reservoir characteristics of Chang 2 reservoir in Area A, which provides a geological basis for the efficient development of oil reservoirs. Comprehensive use of reservoir sandstone thin section identification casting analysis, mercury intrusion analysis, logging analysis and other methods to systematically study the petrological characteristics, pore characteristics and reservoir physical properties of Chang 2 reservoir in Area A. The results show that the reservoirs in the study area are dominated by fine-grained sandstones, with low component maturity and high structural maturity. They are all medium-low porosity, low-permeability and ultra-low permeability reservoirs. Primary intergranular pores and residual intergranular pores are developed, the reservoir drainage pressure is low, which is good-medium, and the mercury removal efficiency is high, indicating that the reservoir has good storage performance and seepage properties.
\end{abstract}

\section{Introduction}

Reservoir is one of the main controlling factors affecting the distribution of oil and gas, and it is the basis for finding remaining oil and developing oilfields [1-3]. Therefore, it is very important to conduct in-depth research on reservoir characteristics and comprehensive evaluation of reservoirs.

The Ordos Basin is a large-scale multi-cycle craton basin. The main oil-bearing layer of the Triassic Yanchang Formation is divided into 10 oil-bearing groups according to sedimentary cycles [4-6]. The Chang 2 reservoir in the study area mainly developed delta plain deposits, mainly sandstone deposits, with the characteristics of multi-stage channel superimposed development. Reservoir sand bodies are controlled by the distribution of sedimentary facies belts, with large oil layers and shallow burial. Chang 2 reservoir is the main production layers in Area A. This study used reservoir sandstone thin section identification, casting analysis, electron microscope scanning, mercury intrusion analysis methods to comprehensively evaluate the petrological characteristics and reservoir characteristics of Chang 2 reservoir in Area $\mathrm{A}$ area, and provide a geological basis for the efficient development of oil reservoirs.

\section{Reservoir petrological characteristics}

Through the identification and statistics of rock flakes, combined with observation under a scanning electron microscope, the petrological characteristics of the reservoirs in the study area were studied in detail, and the rock types were classified based on the three-terminal components of quartz, feldspar, and cuttings ( figure 1).

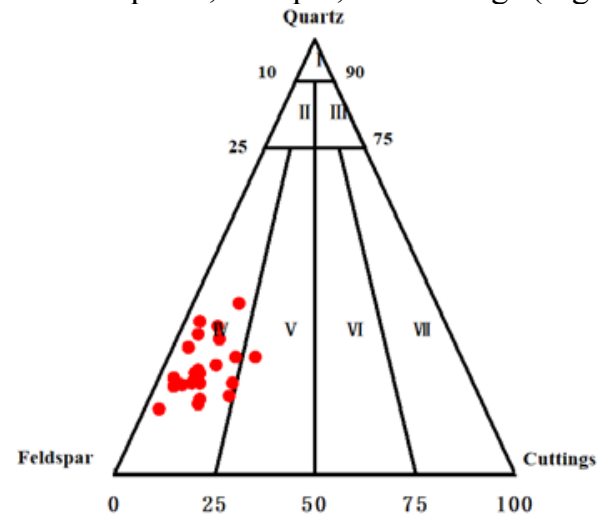

I quartz sandstone, II feldspar quartz sandstone, III lithic quartz sandstone, IV feldspar sandstone, V lithic feldspar sandstone, VI feldspar lithic sandstone, lithic sandstone.

Figure 1. Triangular diagram of Chang 2 rock types in Area A

\subsection{Sandstone characteristics}

The mineral composition maturity of the Chang 2 reservoir rock is relatively low. Statistics show that the clastic composition is dominated by feldspar, with a content of $46.3 \% \sim 48.3 \%$, with an average of $47.2 \%$. Followed by quartz, with a content of $28.6 \% \sim 29.6 \%$, with an average of $29.1 \%$. The content of biotite and cuttings is relatively low, with an average content of 7.3\% and $3.1 \%$, respectively. The composition of the cuttings is mainly acid extruded rock, granitic rock and dynamic metamorphic rock, and mica is mostly chlorite(figure 2).

* Corresponding author: 20190100@xijing.edu.cn 


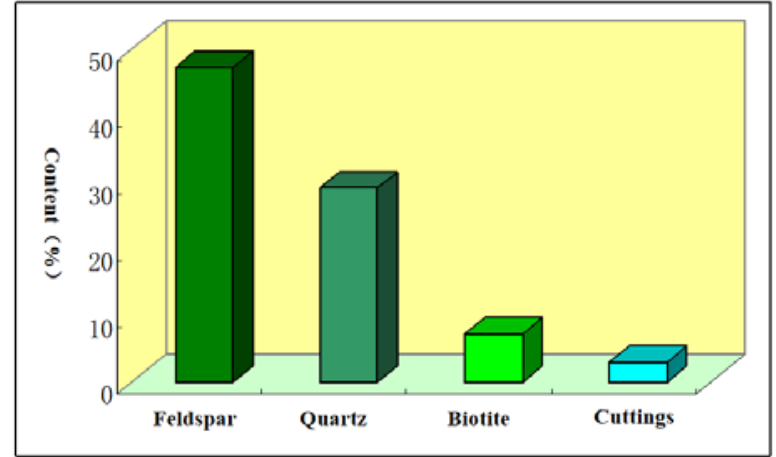

Figure 2. Distribution diagram of rock composition of Chang 2 in Area A

\subsection{Clay mineral characteristics}

The clay minerals in the Chang 2 reservoir in Area A can be divided into two types, the original cause and the autogenous cause. The microscopic characteristics of clay minerals are: primary clay minerals are mostly filled in the pores between the particles, and there is no fresh crystal form. The microscopic characteristics of authigenic clay minerals are more complex and the crystal shape is relatively complete. According to statistics, the absolute content of clay minerals in Chang 2 oil layer in this area is $4.4 \%$ to $10.8 \%$, with an average of $8.4 \%$. There are four main types of clay minerals, namely chlorite, illite, kaolinite, and illite/smectite layers. Among them, chlorite is the main type, with a relative content of $57.5 \%$ to $90.7 \%$ of the total clay minerals, with an average of $74.2 \%$. Observed under a scanning electron microscope, chlorite is mostly distributed vertically on the surface of detrital particles in needle-like or rose-like shapes, and is produced in a pore-filling or cushioning type (Figure 3). The second is kaolinite, with a content of $8.2 \%$ to $18.8 \%$, with an average of $12.7 \%$. It is mostly produced in the form of pages or worms in the reservoir, but its distribution varies greatly in different parts. The content of illite in the reservoir is relatively high, ranging from $4.1 \%$ to $11.7 \%$, with an average of $7.6 \%$. Most of the illite is produced in the pores in the form of filaments, bridges or flakes, blocking the throat. The content of clay minerals in the Iraqi/Mongolian mixed layer is the lowest, with a content of $3.6 \%$ to $6.7 \%$.

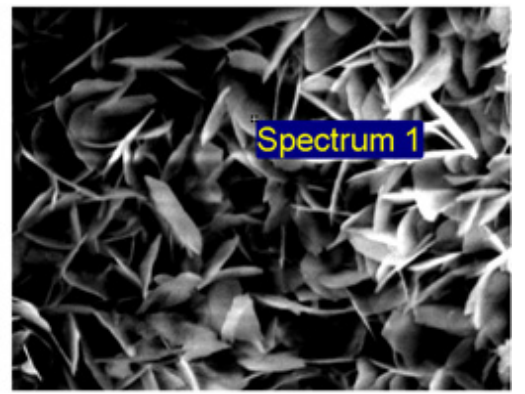

Figure 3. Scanning electron microscope photo of chlorite clay minerals in Chang 2 oil layer in Area A

\section{Reservoir pore type and pore structure}

\subsection{Pore types}

Pore types can be divided into three categories: primary pores, secondary pores and micro-fractures according to their genesis. Primary pores mainly refer to the intergranular pores of detrital particles, and also include interlayer pores and pores. Secondary pores refer to the pores and fractures in the rock after the formation of sedimentary rocks due to leaching, dissolution, metasomatism, dissolution and recrystallization. During the diagenesis process, the primary pores will gradually decrease through compaction, cementation and pressure dissolution. At the same time, the soluble detrital particles and easily soluble formations will dissolve and metasomatize as the buried depth increases, thereby contributing to Development of secondary pores in clastic rocks.

The Chang 2 oil layer group of Yanchang Formation in Area Area A is shallower, and the compaction and pressure solution are weakened, and the primary pores are mainly developed. The primary intergranular pores remaining after the secondary enlargement of mechanical compaction of feldspar and quartz and a variety of cementation are also called residual intergranular pores. Including the residual intergranular pores after the early film-like chlorite and illite cementation, the residual intergranular pores after the secondary growth of quartz and feldspar, and the residual intergranular pores after the filling and cementation of calcite and clay minerals (Figure 4).

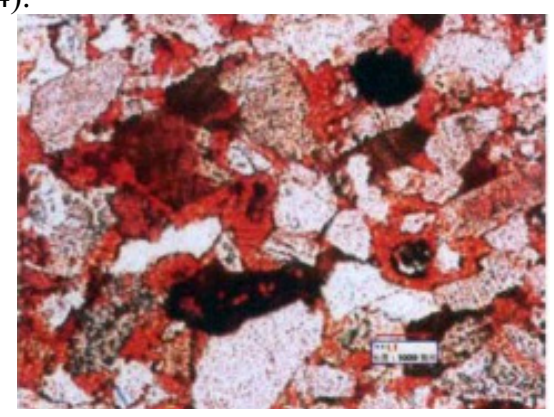

A.Primary intergranular pores

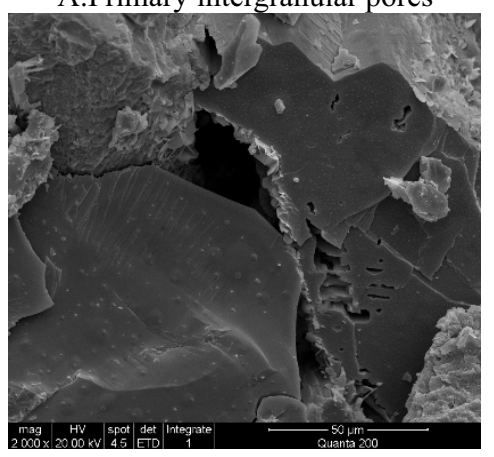

B.Residual intergranular pores

Figure 4. Primary pores in Chang 2 reservoir of Well B95 in Area A 


\subsection{Pore structure characteristics}

In this study, mercury intrusion method was selected to study the pore structure. The capillary pressure curve reflects the size of the pores through which mercury may enter the throat and the volume of pores connected by this throat under a certain displacement pressure. The capillary pressure curve is not only a function of the pore size distribution and pore volume, a function of the pore-throat connection method, but also a function of porosity, permeability and saturation $[7,8]$. The capillary pressure curve is used to characterize the pore structure, the length of the relatively flat section in the middle of the capillary pressure curve indicates the concentration of rock throat distribution and the quality of the sorting. The longer the flat section, the more concentrated the distribution of rock throats, the better the sorting, and the better the flat section. Lower, the larger the radius of the rock throat, the lower the displacement starting pressure, and the better the rock permeability. On the contrary, the higher the gentle section, the smaller the radius of the rock throat, the higher the displacement starting pressure, and the worse the rock permeability.

According to the capillary pressure curve and mercury injection curve data, it can be seen that the displacement pressure, the saturation median pressure, and the median throat radius of the Chang 2 reservoir in the study area have not changed much. The average displacement pressure is $0.19 \mathrm{MPa}$, the average saturation median pressure is $3.41 \mathrm{MPa}$, the average throat median radius is $0.24 \mu \mathrm{m}$, the average mercury removal efficiency is $39.98 \%$, and the average maximum mercury saturation is 93.46\%. In general, the Chang 2 reservoir in Area A area has low displacement pressure and high mercury removal efficiency, indicating that the reservoir has good reservoir and permeability properties.

\section{Reservoir physical properties}

\subsection{Classification standards for reservoir physical properties}

According to the reservoir classification standard in the petroleum industry standard, the physical properties of sandstone reservoirs are divided into 5 types of reservoirs, as shown in Table 1.

Table 1. Classification standards for physical properties of sandstone reservoirs

\begin{tabular}{|c|c|c|}
\hline Classification & $\begin{array}{c}\text { Porosity } \Phi \\
(\%)\end{array}$ & $\begin{array}{c}\text { Permeability K } \\
\left(\times 10^{-3} \mu \mathrm{m}^{2}\right)\end{array}$ \\
\hline Extra High & $\Phi \geq 30$ & $\mathrm{~K} \geq 1000$ \\
\hline High & $25 \leq \Phi<30$ & $500 \leq \mathrm{K}<1000$ \\
\hline Medium & $15 \leq \Phi<25$ & $50 \leq \mathrm{K}<500$ \\
\hline Low & $10 \leq \Phi<15$ & $5 \leq \mathrm{K}<50$ \\
\hline Extra Low & $\Phi<10$ & $\mathrm{~K}<5$ \\
\hline
\end{tabular}

\subsection{Reservoir physical characteristics}

According to the logging data, the porosity and permeability of the studied well sections are statistically classified. The results show that the overall physical properties of the Chang $2_{1}$ layer are better than those of the Chang $2_{2}$ layer, which may be related to the compaction of the formation. The maximum permeability varies greatly between each small layer, especially the difference between the maximum permeability of the Chang $22_{2}{ }^{1}$ and Chang $22^{3}$ layers Nearly 10 times. On the one hand, there may be a large difference in the cored samples, and the Chang $22^{3}$ layer has less physical property data, which cannot fully represent the real state of the physical properties of the layer. On the other hand, there may be deviations in logging data or calculation methods. Lead to the emergence of undue real data. However, there are more than 60 data points in the physical property statistics of each sub-layer, and more than 200 data points in individual layers. However, there are only 1 to 2 suspected under-real data obtained by statistics, which account for a small proportion in the overall sample. can be ignored. Therefore, the physical property data of each sublayer of Chang 2 in this statistics can basically reflect the physical property characteristics of the formation.

After sub-layer statistics of physical property data, it is found that the average porosity of each sub-layer is greater than $10 \%$, the average permeability is less than $50 \times 10^{-}$ ${ }^{3} \mu \mathrm{m}^{2}$, and the physical property classification of the entire Chang 2 formation has little difference. Therefore, in this study, all the physical property data of Chang 2 formation were calculated according to the physical property classification standard, and the frequency distribution histogram of porosity and permeability (Figure 5, Figure 6) was drawn to grasp the change of Chang 2 physical property from a macro perspective feature.

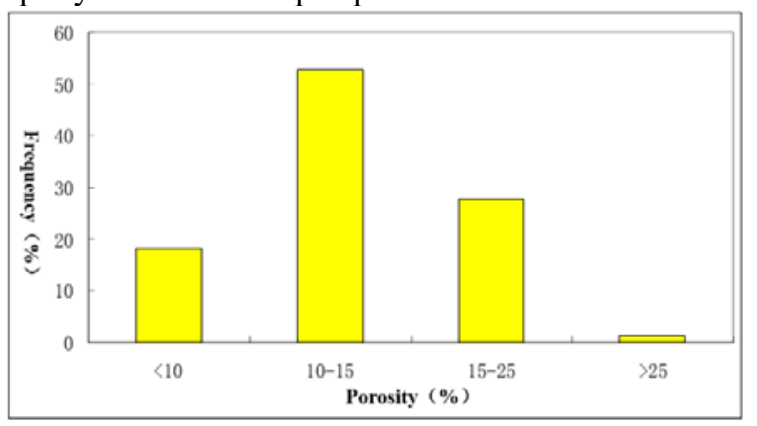

Figure 5. Porosity distribution histogram of Chang 2 reservoir in Area A

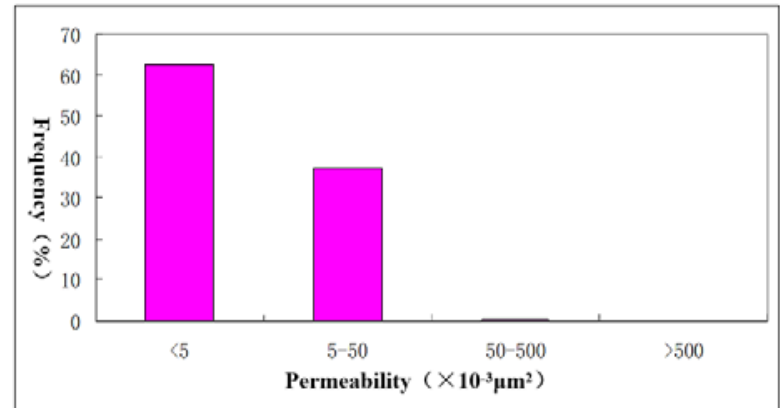

Figure 6. Histogram of permeability distribution of Chang 2 reservoir in Area A 
From the above two physical property distribution histograms, it can be seen that the porosity of the Chang 2 formation in Area A is mainly $10-15 \%$, followed by $15-$ $25 \%$, and the proportion of porosities less than $10 \%$ and greater than $25 \%$ is relatively small. Most of the permeability is less than $5 \times 10^{-3} \mu \mathrm{m}^{2}$, followed by $5 \sim$ $50 \times 10^{-3} \mu \mathrm{m}^{2}$, and there is almost no data with permeability greater than $50 \times 10^{-3} \mu \mathrm{m}^{2}$. According to the reservoir physical property classification standard, the Chang 2 reservoir is a medium-low porosity, low-permeability, and ultra-low permeability sandstone reservoir.

\section{Conclusion}

1. Chang 2 in Area Area A is dominated by fine-grained sandstone, and the compositional maturity of the reservoir sandstone is low. The clastic composition is dominated by feldspar, followed by quartz, and the content of rock fragments is low. Reservoir sandstone has low component maturity and high structural maturity.

2. The Chang 2 oil layer of Yanchang Formation in the study block is shallower, the compaction and pressure solution are weakened, and the primary intergranular pores and residual intergranular pores are mainly developed. The reservoir drainage pressure is low, the mercury removal efficiency is high, indicating that the reservoir has good reservoir and permeability properties.

3.The porosity of Chang 2 formation is mainly $10-15 \%$, and the permeability is mostly less than $5 \times 10^{-3} \mu \mathrm{m}^{2}$. According to the classification standard of reservoir physical properties, the Chang 2 reservoir is a mediumlow porosity, low permeability, and ultra-low permeability sandstone reservoir.

\section{References}

1. Zhao Jingzhou, Wang Yongdong, Meng Xiangzhen. Distribution law of Triassic Chang 2 reservoir in the eastern part of the northern slope of Shaanxi, Ordos Basin[J]. Petroleum Exploration and Development 2007(1):23-27.

2. Guo Yanqin, Study on the micro characteristics of Yanchang Formation reservoir in Fuxian exploration area[D]. Northwest University, 2006.

3. Liu Chunyan, Wang Yi. Sedimentary characteristics and physical properties of Yanchang Formation in Fuxian area, Ordos Basin[J]. World Geology, 2009,28(4):23-26.

4. Xu Jianhong.Geological characteristics of the Triassic Yanchang Formation reservoir in Chenglinsong Ordos Basin[J]. Journal of Southwest Petroleum University,2007,29(5)13-17.

5. Wu Fuli, Li Wenhou, Li Yuhong. Delta deposition and evolution of the Upper Triassic Yanchang Formation in the Ordos Basin[J]. Journal of Palaeogeography,2004(3 ):307-315.

6. Wang Kai, Wu Fuli, Wang Erhu, etc. Reservoir characteristics analysis of Chang 2 oil layer group in Zichang Oilfield[J]. Journal of Yangtze University,
2013, 10(8):29-30.

7. Guo Yanqin. Study on the micro characteristics of Yanchang Formation reservoir in Fuxian exploration area[D]. Northwest University, 2006.

8. Wang Feng. Study on the sedimentary, sequence evolution and lithologic reservoir characteristics of the Triassic Yanchang Formation in the Ordos Basin[D]. Chengdu University of Technology, 2007. 